\title{
Ambiente de telediagnóstico colaborativo utilizando plataforma inteligente de auxílio à tomada de decisão
}

\author{
Gláucia Regina Medeiros Azambuja Sizilio*, Cicília Raquel Maia Leite, Ana Maria Guimarães Guerreiro, \\ Adrião Duarte Dória Neto
}

Resumo Em função de dispersão geográfica (por se encontrarem em diferentes hospitais, cidades ou países) e/ou problemas de conciliação de tempo, os profissionais da área de saúde (médicos e outros) têm dificuldade de sincronização espaço-temporal para realização de trabalho conjunto. Entretanto, existe uma crescente demanda para a realização de trabalho colaborativo envolvendo vários profissionais para análise da situação de um paciente ou sua confirmação (diagnóstico ou segunda opinião). Por outro lado, também é crescente a demanda pela utilização de inteligência computacional no apoio à tomada de decisão na área de saúde. Inserindo-se nesse contexto, o objetivo deste trabalho foi o desenvolvimento de um ambiente de telediagnóstico colaborativo, com a incorporação de recursos de inteligência computacional em módulos especialistas responsáveis pela análise de exames e dados clínicos específicos do paciente, visando prover um pré-diagnóstico. O modelo desenvolvido, operando tanto de forma síncrona (usuários conectados no mesmo instante de tempo) quanto assíncrona (usuários interagindo em momentos distintos), é capaz de: i) prover o suporte ao trabalho colaborativo a distância de múltiplos usuários (equipe médico-hospitalar); ii) disponibilizar aos usuários informações gráficas e textuais; e iii) incorporar módulos inteligentes que, utilizando técnicas de inteligência computacional, são capazes de realizar pré-diagnósticos a partir de dados e exames de pacientes.

Palavras-chave Telemedicina, Telediagnóstico, Trabalho colaborativo a distância, Inteligência computacional, Sistema de apoio à decisão, Automação hospitalar.

\section{Collaborative remote diagnostics environment using intelligent platform support decision}

\begin{abstract}
Due to geographical dispersion (for without are in different hospitals, city or country) and/or time problems, health professionals, particularly physicians, have difficulty synchronizing space and time to perform joint work. However, there is growing demand for collaborative work involving several professionals to analyze the situation of a patient or its confirmation (diagnosis or second opinion). On the other hand, there is also a growing demand for the use of computational intelligence to support decision making in health care. In that context, the objective of this work is to develop a collaborative environment for telediagnosis, with the incorporation of computational intelligence resources into specialist modules responsible for testing the patient's exams and specific clinical data, to provide a pre-diagnosis. The model developed, operating both synchronously (users connected at the same instant of time) or asynchronously (users interacting at different times), is capable of: i) provide support for collaborative work away multiple users (healthcare); ii) provide users with graphical and textual information; and iii) incorporate specialistas modules using computational intelligence techniques, are able to perform pre-diagnostics from data and examinations of patients.
\end{abstract}

Keywords Telemedicine, Remote diagnostics, Remote collaborative work, Computational Intelligence, Decision support system, Hospital automation. 


\section{Extended Abstract}

\section{Introduction}

At the same time it develops and specializes rapidly, the health sector faces the difficulty of space-time synchronization among professionals, sometimes provide detrimental to diagnosis and second opinions.

This paper presents a model of intelligent virtual environment for medical interaction in order to carry out telediagnosis to help decision making. The intelligent platform is composed of Pre-Diagnosis Modules (MPD) that are responsible for testing the patient's exams and specific clinical data, using resources of Computational Intelligence (CI).

\section{Material and Methods}

The model of collaborative remote diagnostics environment using intelligent platform support decision, consolidated through Intelligent Virtual Environment of Medical Interaction (named AVIIM), object of the present study, allows the realization of telediagnosis and second opinion, providing the following main services: access to data and information on clinical cases including text, images and videos; realization of pre-diagnosis through integrated intelligent expert modules; availability of synchronous and asynchronous tools that enable interaction between doctors through texts, audioand/or image; availability of tools for carrying out cooperative work through texts or images; constantly updated database with the record of synchronous and asynchronous activities performed; updating of institutions, physicians and patients registration data; history storage and final results (decision and procedures) of clinical cases.

In AVIIM, the integrated unit of work (performed by medical staff in a cooperative way), is the clinical case of one patient that, through interactions made and inferences required, result in partial and final diagnosis issued by medical staff, archived and available by AVIIM according to interaction with the environment as shown in Figure 1.

The data, stored on remote server, are available to all participants who access the virtual environment and interact through synchronous and asynchronous tools of the environment. In addition, any participant may require pre-diagnosis of intelligent modules built into AVIIM.

The proposed model combines a set of interactive features inherent in virtual environments, incorporating intelligent modules that perform pre-diagnostic analysis of specific exams (like: FNAFine Needle Aspirate of breast tissue; and skin tissue biopsy), involving the following parts: the clinical case under discussion; physicians (team coordinator and participants) geographically and/or temporally dispersed; health professionals responsible for integration of patient's data and examination results (data, image and / or video) or any person invited to join the team for the diagnosis; Pre-Diagnostic Modules (MPD); and resources for collaborative work at a distance (as for example: Forum; wiki; and tool award and procedures). The AVIIM also applies to procedures for second opinion in which a specialist may request others doctors opinion and/or MPD opinion.

It is noteworthy that the AVIIM allows patient data to be viewed in a transparent way by physicians regardless of where each professional is located, the time when he accesses the environment and where the data is stored.
The architecture proposed in this paper (Figure 2) is based on the use of intelligent systems techniques, on the acquisition and data processing and on the information processing and execution of distance cooperative work for the diagnosis achievement. The prototype has been developed on the JEE 6.0 platform, depending on the availability of open-source implementations for it, as well as being specified in the Java language, which allows easy integration with MatLab ${ }^{\circledR}$, language chosen for the creation of the first MPD, which were: 1) Fine Needle Aspirate of breast tissue, conducting pre diagnosis from the analysis of cell nucleus using Fuzzy logic; 2) vital signs in AVC, performing pre diagnosis of ICU patients with clinical picture of Cerebral Vascular Accident, using Fuzzy logic; and 3) skin tissue biopsy, which performs the analysis and classification of skin lesions using Support Vector Machines.

\section{Results and Discussion}

The two main system interfaces are: Clinical Case Installation and Clinical Case Discussion.

The Clinical Case Installation interface (Figure 3) consists of sub-modules presenting two examples of internal level screen: Set sessions; and Edit Sessions.

To install a clinical case the medical coordinator can follow the flow diagram in Figure 4.

The Clinical Case Discussion interface (Figure 5), is composed of sub-modules and the coordinator can create two sessions to begin the work as shown.

With respect to resources that AVIIM provides in this interface to publish, interact and evaluation, the medical coordinator has a large number and variety of tools, which are: providing information and scheduling activities: simple text pages, HTML pages; access to files in any format (pdf, doc, ppt, flash, etc.) or external links (URLs), access to directories (file folders on the server), labels, interactive activities with AVIIM (Ex: prediagnosis request to a specialist module); eBooks, glossaries, questions, images analysis with synchronous and asynchronous interactivity, and questionnaires, chats, webconference, wiki; discussion forum; journals; file; mural. Tools for monitoring the participants are also available: access assessment; and participation assessment.

The flowchart of functioning of AVIIM, in view of participating physicians and considering a clinical case installed for diagnosis is shown in Figure 6.

\section{Conclusion}

The desenvolved environment is a computing solution capable of supporting the medical diagnosis, assisting decision-making from collaborative work and using intelligent systems techniques. The use of AVIIM can provides the realization of remote diagnostics by medical or multidisciplinary teams geographically and / or temporally distant, and providing them access to information that is usually not available.

The main contributions of this paper are: a virtual environment of medical interaction model, customized to realize telediagnosis and second opinion; the main interfaces (Clinical Case Installation and Clinical Case Discussion); and the incorporation of intelligent components in a virtual environment to help medical telediagnosis. 


\section{Introdução}

A área da saúde, ao mesmo tempo em que se desenvolve e especializa aceleradamente, enfrenta dificuldade de sincronização espaço-temporal entre os profissionais. A dispersão geográfica dos médicos e/ou a dificuldade na conciliação de tempo para realizar trabalhos conjuntos em um mesmo instante, por vezes prejudica a realização de diagnósticos e a de segundos pareceres.

$\mathrm{O}$ grande volume e variedade das informações envolvidas, bem como as especificidades dos processamentos relacionados ao trabalho colaborativo a distância, essenciais ao apoio à tomada de decisão, tornam bastante complexo o controle, o tratamento e o gerenciamento dos dados e das ações que subsidiam os processos de telediagnóstico.

Pesquisas voltadas à automatização de ambientes hospitalares relacionadas ao diagnóstico têm sido desenvolvidas, abordando vários dos problemas pertinentes aos processos que podem ser otimizados. Exemplificando, pode-se citar trabalhos voltados para o desenvolvimento de ambientes virtuais colaborativos para diagnóstico ou consulta no campo da telemedicina (Barbosa et al., 2003; Krsek et al., 2009; Soares, 2002) e trabalhos relacionados ao desenvolvimento de sistemas inteligentes aplicados ao diagnóstico na área de saúde (Leite et al., 2010; Soares, 2008; Sriraam et al., 2008).

Os ambientes virtuais colaborativos citados permitem a realização de trabalho cooperativo a partir da troca de informações. A forma mais comumente utilizada é o envio de informações do paciente, pela internet ou por meio de intranet, para um médico distante geograficamente, que analisa as informações e emite um diagnóstico, enviado ao médico de origem pelo mesmo ambiente. Algumas propostas contemplam o tráfego de imagens e vídeos e a possibilidade de mais de um médico dar a sua opinião. Por sua vez, os trabalhos relacionados ao desenvolvimento de sistemas inteligentes aplicados ao diagnóstico tratam de aplicações que utilizam técnicas de inteligência computacional na realização de pré-diagnósticos específicos, mas de forma presencial, disponibilizados no ambiente de trabalho do médico ou no local onde se encontra o paciente.

Neste trabalho, apresenta-se um modelo de ambiente de telediagnóstico colaborativo utilizando plataforma inteligente de auxílio à tomada de decisão. A plataforma inteligente é composta de Módulos de Pré-diagnóstico (MPD) que são responsáveis pela análise de exames e dados clínicos específicos do paciente, utilizando recursos de Inteligência Computacional (IC).

\section{Materiais e Métodos}

\section{Telemedicina - Telediagnóstico}

Visando propiciar maior segurança, confiabilidade e robustez aos serviços e procedimentos, principalmente por lidar com vidas humanas, a área de saúde vem informatizando seus processos.

As principais referências estão orientadas a: sistemas de monitoramento (materiais, funcionários e pacientes); sistemas de gerenciamento e controle (marcação de consulta, prontuário eletrônico, laboratoriais, entre outros); sistemas de comunicação (rastreamento de pacientes, materiais etc); equipamentos/dispositivos médicos, hospitalares e de laboratórios; e sistemas de apoio à decisão e/ou ao trabalho colaborativo (por especialidade) (Hovenga, 2010).

Dentre os desafios mais recentes desta informatização encontra-se a disponibilização de serviços e o desenvolvimento de atividades médicas de forma remota (a distância), caracterizando, pois, a Telemedicina. A Organização Mundial de Saúde (2009) define que Telemedicina compreende a oferta de serviços ligados aos cuidados com a saúde, nos casos em que a distância é um fator crítico; tais serviços são prestados por profissionais da área, em estabelecimentos de saúde, usando Tecnologias da Informação e Comunicação (TIC) para o intercâmbio de informações válidas para diagnósticos, prevenção e tratamento de doenças e a contínua educação de prestadores de serviços em saúde, assim como para fins de pesquisas e avaliações.

As aplicações da telemedicina podem ser classificadas em cinco tipos: telemonitoramento, teleterapia, teledidática, telefonia social e telediagnóstico (Feng, 2007; Shen, 2007).

O telediagnóstico, objeto deste trabalho, caracteriza-se pelo envio remoto de dados, desde sinais vitais a dados laboratoriais e imagens médicas, como também realização de consultas remotas ao paciente, para fins de tomada de decisão diagnóstica entre especialistas médicos. Nesta área podem ser vislumbrados os cenários de diagnóstico remoto cooperativo e de segunda opinião, que é uma consulta adicional com outro médico ou grupo de médicos, solicitada pelo paciente ou pelo médico que o está atendendo, com o objetivo de garantir a corretude de um diagnóstico.

As atividades de segunda opinião estão se tornando cada vez mais importantes devido ao nível crescente de conscientização e de conhecimento dos pacientes a respeito de seus problemas de saúde; ao nível de complexidade científica e técnica da medicina; e 
também aos problemas legais e econômicos associados à prática médica. Para o paciente, uma segunda opinião resulta em uma menor probabilidade de erros no diagnóstico médico, mas pode também provocar um aumento do custo e do tempo de espera para a obtenção de um diagnóstico. A segunda opinião é também uma solução tipicamente aplicada pelos planos de saúde privados para se certificar da necessidade de cirurgias complexas no tratamento de pacientes e reduzir custos (Sabbatini, 2010).

\section{Ambientes para trabalho colaborativo à distância}

A interatividade, essencial à realização de trabalho colaborativo, é compreendida como a possibilidade de intercambiar saberes, rompendo com a unidirecionalidade dos processos comunicacionais, promovendo uma interação simultânea que enfatiza os aspectos quantitativos (pessoas interagindo) e principalmente qualitativos, como variedade, riqueza e natureza das interações (Paulon et al., 2009). Os ambientes virtuais propiciam a interatividade e contribuem com uma extraordinária expansão da informação, do conhecimento e com a diminuição das distâncias (European..., 2009).

Um ambiente virtual é uma localidade da rede mundial de computadores, onde a interação entre os participantes não precisa mais de lugar e nem de tempo determinados, podendo acontecer a qualquer hora, por meio de recursos de gerenciamento e disponibilização de informações e ferramentas que atuam como mediadores do processo de construção colaborativa, como disponibilização de material (textos, imagens e vídeos), salas de conversação, fóruns de discussão, envio de mensagens, questionários, wikis, blogs etc. Ambientes virtuais que permitem a realização de trabalho colaborativo disponibilizam duas formas de comunicação para promover e desenvolver a interatividade entre os participantes: síncrona e assíncrona.

$\mathrm{Na}$ forma síncrona (que ocorre em tempo real - on-line), as partes se comunicam de modo instantâneo, devendo acessar o ambiente ao mesmo tempo, independente do lugar onde estejam. Nela ocorre a sensação de agilidade no desenvolvimento dos trabalhos, provocada pelas características desse tipo de comunicação (Encyclopedia..., 2009). Algumas características da comunicação síncrona são: comunicação espontânea; resposta espontânea; motivação (sinergia nos trabalhos individuais e em grupo, motivando os participantes a criarem e participarem das atividades); presença (fortalece o sentimento de comunidade); feedback em tempo real (o rápido retorno fomenta o desenvolvimento das atividades); ritmo (ajuda aos participantes a serem criativos).

$\mathrm{Na}$ forma assíncrona (que está desconectada do tempo e do espaço), cada participante pode atuar em função do seu tempo disponível, sendo mais confortável em relação às disponibilidades e necessidades de interação efetiva (Encyclopedia..., 2009). Algumas características da comunicação assíncrona são: flexibilidade temporal (acesso às atividades a qualquer hora); tempo para reflexão (o tempo poderá ser otimizado para estudos e reflexão sobre o material apresentado para discussão, para ter ideias e preparar os retornos, para checar as referências utilizadas e possibilidade de acesso ao material quantas vezes for necessário); facilidade de participação (possibilita a administração da realização das atividades de forma a aproveitar todas as oportunidades de tempo, podendo ocorrer a integração de ideias e discussão sobre o assunto em fóruns específicos).

O nível de sucesso da interatividade que acontecerá no trabalho colaborativo dependerá da escolha que for feita entre essas duas formas de comunicação, considerando que ambas têm características específicas, devendo ser utilizadas forma adequada e harmônica.

\section{Inteligência computacional}

A Inteligência Computacional (IC) possibilita, através de técnicas inspiradas na natureza, o desenvolvimento de sistemas inteligentes que imitem aspectos do comportamento humano, tais como: aprendizado, percepção, raciocínio, evolução e adaptação. Alguns exemplos de técnicas de Inteligência Computacional são: Redes Neurais, inspirada em neurônios biológicos; Computação Evolucionária, inspirada em evolução biológica; Lógica Fuzzy, inspirada em processamento linguístico e Sistemas Especialistas, inspirada em processo de inferência (Engelbrech, 2007).

O processo de diagnóstico, realizado por profissional médico, habitualmente analisa os órgãos ou a região anatômica afetada, as funções comprometidas e as causas dessas alterações, além da faixa etária, estilo de vida e situação geográfica do paciente. Quando não se trata de algo facilmente definido, o médico utiliza um raciocínio lógico dedutivo, buscando encontrar padrões de apresentação clínica compatíveis com diagnósticos mais prováveis, onde cada nova informação ou sintoma pode confirmar uma suspeita e eliminar outra. O médico trabalha com sistemas de reconhecimento, fazendo suposições baseado no que é estatisticamente provável para determinada situação. Nesta ótica, o telediagnóstico utilizando técnicas de sistemas inteligentes aborda não só a identificação direta de uma patologia, mas o faz com 
base em uma visão consequente, provendo um suporte à decisão médica detalhado e com grande quantidade de informações processadas. O telediagnóstico, neste estudo, leva em conta as tecnologias baseadas nos princípios de IC.

O modelo do ambiente de telediagnóstico colaborativo utilizando plataforma inteligente de auxílio à tomada de decisão, consolidado através do AVIIM (Ambiente Virtual Inteligente de Interação Médica), objeto de estudo deste trabalho, é apresentado a seguir.

\section{Visão Geral do AVIIM}

O AVIIM possibilita a realização de telediagnósticos e segunda opinião, disponibilizando como principais serviços: acesso a dados e informações sobre casos clínicos, abrangendo textos, imagens e vídeos; realização de pré-diagnósticos por módulos especialistas inteligentes integrados; disponibilização de ferramentas síncronas e assíncronas que possibilitam a interação entre os médicos (textual, por áudio e/ ou por imagem; disponibilização de ferramentas para realização de trabalho cooperativo; atualização constante de banco de dados com registro das atividades síncronas e assíncronas realizadas; atualização de dados cadastrais de instituições, médicos e pacientes; armazenamento da história e diagnóstico final (laudos e procedimentos) dos casos clínicos.

O desenvolvimento e/ou a utilização de aplicações em Telemedicina têm características únicas e particulares, apresentando dificuldades éticas e legais (Sarhan, 2009). Neste sentido, o desenvolvimento do AVIIM segue as normas definidas no manual de certificação para registro eletrônico em saúde da Sociedade Brasileira de Informática em Saúde (Sociedade..., 2009).

No AVIIM, a unidade de trabalho integrado (realizado cooperativamente pela equipe médica) é o caso clínico de um paciente que, através de interações realizadas e inferências requisitadas, resultam em diagnósticos parciais e finais emitidos pela equipe médica, sendo arquivados e disponibilizados pelo AVIIM, conforme interação com o meio externo ilustrada na Figura 1.

Através do AVIIM, via internet, o médico coordenador da equipe: i) introduz no sistema um conjunto de informações do caso clínico, que inclui informações textuais, imagens e/ou vídeos; ii) credencia médicos especialistas e outros profissionais para participarem da equipe que realizará o diagnóstico remoto; iii) inicia os trabalhos colaborativos para realização de telediagnóstico. Os dados ficam armazenados em servidor remoto, estando disponíveis para todos os participantes, que acessam o ambiente virtual e interagem entre si, através de ferramentas síncronas e assíncronas do ambiente. Além disso, qualquer participante pode solicitar pré-diagnósticos de módulos inteligentes incorporados ao AVIIM. Os médicos participantes avaliam, em conjunto e de forma colaborativa, os dados do caso clínico e

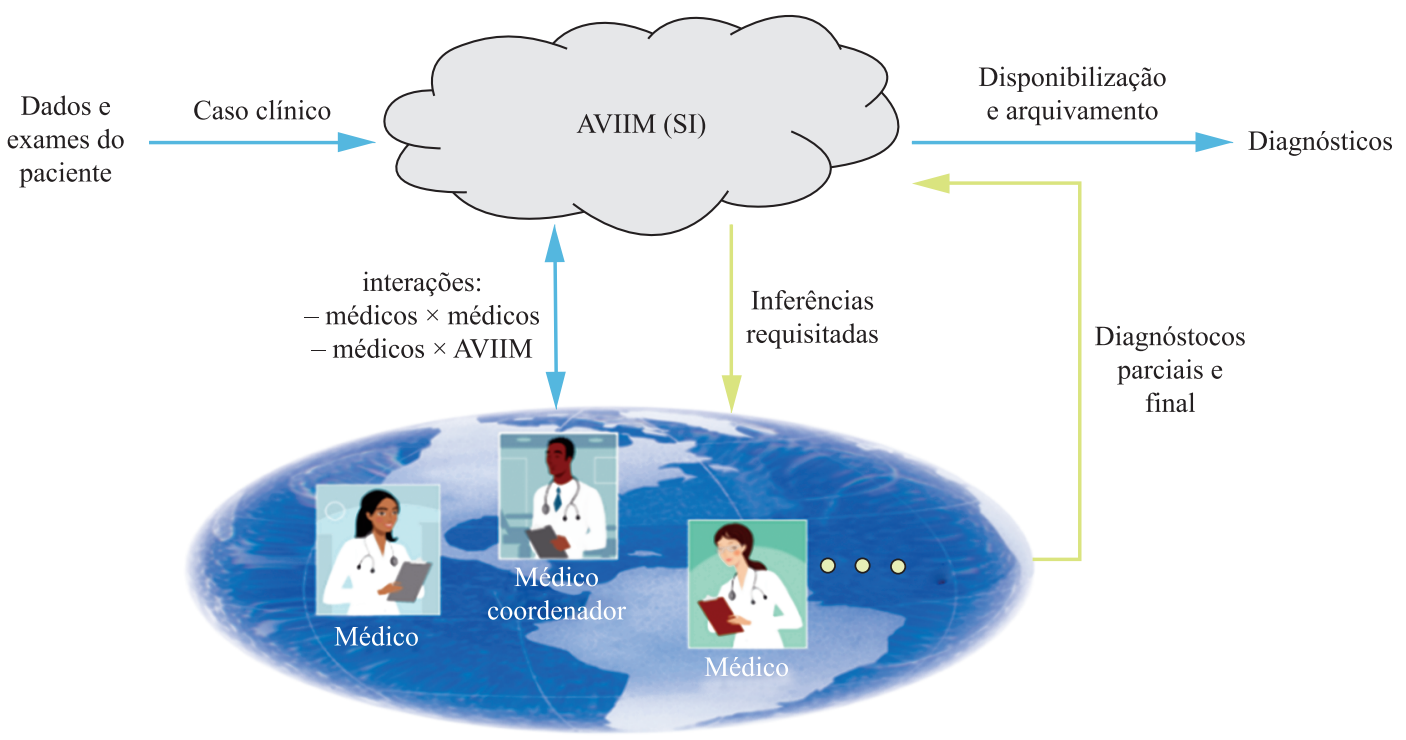

Equipe médica dispersa

Figura 1. Visão geral do AVIIM.

Figure 1. AVIIM general vision. 
emitem pareceres, abrangendo laudo e sugestão de procedimentos, até que a equipe médica chegue a uma conclusão sobre o caso (Figura 1).

O modelo proposto combina um conjunto de recursos interativos inerentes a ambientes virtuais (como, por exemplo: exposição de textos, vídeos e imagens; chat; diário; fórum; mural; glossário; ficheiro; tema; pesquisa de opinião; questionário; tarefa; workshop; wiki; e webconference), incorporando módulos inteligentes que realizam análises pré-diagnósticas de exames específicos (como: FNA - Fine Needle Aspirate de tecido mamário; ECG - Eletrocardiograma; Biópsia de tecico mamário; Biopsia de tecido de pele; e outros), tendo como atores envolvidos: caso clínico que está sendo discutido; médicos (coordenador da equipe e participantes) dispersos geográfica e/ou temporalmente; profissionais da área de saúde, responsáveis pela inserção dos dados dos pacientes e dos resultados dos exames (dados, imagem e/ou vídeo) ou convidados para participar da equipe para realização do diagnóstico; módulos de pré-diagnóstico (MPD); e recursos para realização de trabalho colaborativo a distância (como, por exemplo: fórum; avaliação conjunta de dados e exames do pacientes; wiki; e ferramenta laudo e procedimentos). O AVIIM também é aplicável aos procedimentos de segunda opinião, podendo um médico especialista solicitar a opinião de outro(s) médico(s) e/ou a opinião de MPD.

Ressalta-se que o AVIIM permite que os dados do paciente sejam vistos de forma transparente pelos médicos, independente de onde cada profissional estiver localizado, do momento em que o mesmo acessar o ambiente e de onde estiverem armazenados os dados.

O AVIIM apresenta como principais recursos: módulos inteligentes de inferência, utilizando métodos de classificação e diagnóstico, para auxiliar a tomada de decisão da equipe médica envolvida na realização de diagnóstico; ferramentas necessárias à realização e à coordenação do trabalho colaborativo, que permitam a comunicação síncrona ou assíncrona aos participantes; ferramentas que permitam a inserção, disponibilização e gerenciamento de dados, imagens, vídeos e sons; gerenciamento dos participantes e das atividades na realização de telediagnóstico.

\section{Arquitetura do AVIIM}

A arquitetura proposta neste trabalho é baseada na utilização de técnicas de sistemas inteligentes, na aquisição e tratamento de dados (textuais, imagem e vídeo), no processamento de informação e execução de trabalho cooperativo a distância na realização de diagnóstico (Figura 2).
A arquitetura do AVIIM é composta dos seguintes módulos: área de trabalho, responsável por integrar todos os componentes do ambiente, permitindo o acesso aos mesmos; caso clínico, armazena e acessa as informações relacionadas ao caso clínico, abrangendo o relato, resultados de exames, laudos e procedimentos; interatividade, permite o acesso às atividades síncronas e assíncronas que propiciam a comunicação entre os participantes da equipe e a realização do trabalho colaborativo; módulos de pré-diagnóstico (MPD1, MPD2, ..., MPDn), que utilizam técnicas de sistemas inteligentes, têm processamento independente, disponibilizam prédiagnósticos a partir de solicitação, interagem com entidades externas ou internas na realização de coleta e armazenamento de dados e informações processadas, sendo capazes de capturar, tratar e analisar dados, imagens ou vídeos, dependendo da sua especialidade.

\section{Implementação do AVIIM}

O protótipo foi desenvolvido na plataforma JEE 6.0, em função da disponibilidade de implementações open-source para a mesma, além dela ser especificada em linguagem Java, o que permite uma fácil integração com MatLab ${ }^{\circledR}$, linguagem escolhida para a criação dos primeiros MPDs, que foram: 1) FNA - Fine Needle Aspirate de tecido mamário, realizando pré-diagnóstico a partir da análise dos núcleos celulares, utilizando lógica Fuzzy; 2) Sinais Vitais em AVC, realizando pré-diagnóstico de pacientes internados em UTI com quadro clínico de Acidente Vascular Cerebral (AVC), utilizando lógica Fuzzy no processamento inteligente dos sinais vitais do paciente (monitorados por monitor cardíaco multiparamétrico); e 3) biopsia de tecido de pele, que realiza a análise e classificação de lesões da pele utilizando SVM (Support Vector Machines).

\section{Módulos de pré-diagnóstico (MPD)}

Os módulos inteligentes (MPD), responsáveis pela realização de pré-diagnósticos específicos utilizando técnicas diversas de inteligência computacional, são independentes entre si e, após previamente testados e validados em suas funcionalidades específicas, são integrados ao AVIIM utilizando padrão HL7 (Health..., 2010), visando garantir a interoperabilidade entre estes e o ambiente de telediagnóstico colaborativo. Este projeto de desenvolvimento do AVIIM está vinculado a um grupo de pesquisa, com subgrupos específicos trabalhando no desenvolvimento de cada módulo especialista inteligente, gerando publicações independentes para cada MPD como é o caso de Leite et al. (2010) e Soares (2008). Na atual fase do projeto, prioriza-se o desenvolvimento e/ou a incorporação de módulos inteligentes especializados 


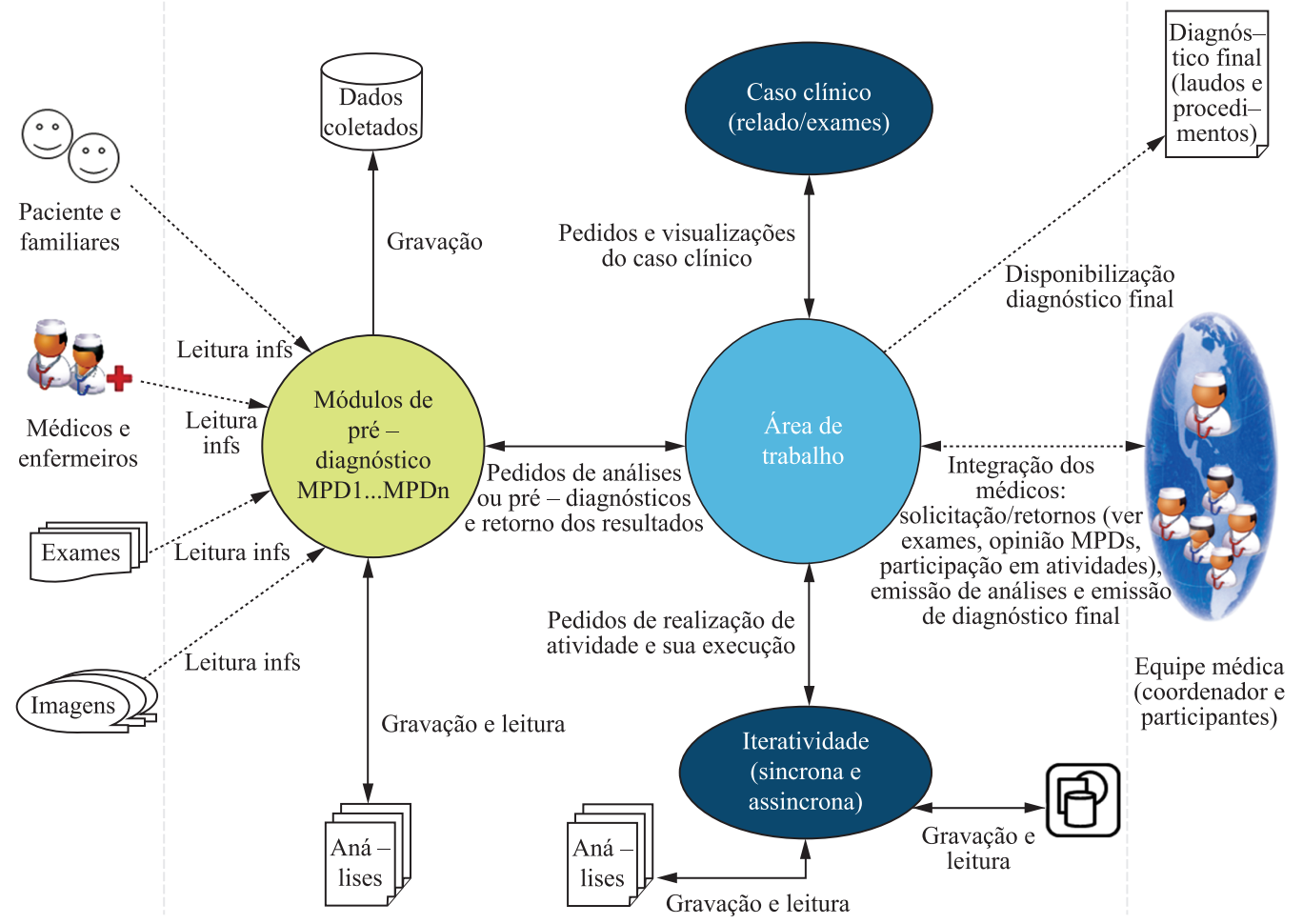

Figura 2. Arquitetura do AVIIM.

Figure 2. AVIIM architecture.

em pré-diagnóstico de câncer (mama, pele, próstata, laringe e outros).

Um exemplo de validação foi a realizada no MPD "Sinais Vitais em AVC", onde, para a medida deste resultado, foram disponibilizadas 100 (cem) inferências por meio de formulários para que os médicos envolvidos pudessem validar o monitoramento e processamento inteligente dos sinais vitais disponibilizado pela arquitetura proposta e pelo modelo fuzzy. As análises foram realizadas mediante respostas dos formulários disponibilizados por três médicos e discussões dos resultados. Obteve-se das 100 inferências: 92\% com diagnósticos semelhantes e $8 \%$ com diagnósticos diferentes. Após discussão dos resultados com os especialistas verificou-se que dos $8 \%$ com diagnósticos diferentes: 4\% o MPD inferiu de maneira correta; e $4 \%$ eles divergiram entre si.

Outro exemplo de validação foi a realizada no MPD "FNA tecido mamário", que foi aplicado à base de dados "padrão ouro" WDBC - Breast Cancer Wisconsin Diagnostic (obtida a partir do repositório da UCI - University of California Irvine), contendo imagens de biópsias de tecidos mamários coletados por FNA. Por meio de validação cruzada, obteve-se das 569 inferências realizadas: 98,11\% de sensibilidade (tumor maligno classificado corretamente) e $85,15 \%$ de especificidade (tumor benigno classificado corretamente).

\section{Validação do AVIIM}

A validação do ambiente de trabalho colaborativo AVIIM, realizada em 4 etapas, abrange:

- Etapa 1: validação da especificação do sistema - revisões dos requisitos em reuniões realizadas com médicos especialistas (cardiologia, clínica médica e patologia);

- Etapa 2: validação da arquitetura do ambiente - revisões de projeto;

- Etapa 3: validação da implementação do ambiente - análises das funcionalidades e das interfaces, realizadas com a participação de médicos especialistas (cardiologia, mastologia, clínica médica e patologia);

- Etapa 4: validação final do ambiente - testes do produto em situação real, analisando (por meio do preenchimento de formulário eletrônico) aspectos de tempo, funcionalidade, interatividade e outros, com a participação de médicos especialistas (cardiologia, mastologia, neurologia, clínica geral e patologia) dispersos geográfico e/ou temporalmente, que acessaram 
o AVIIM de seus computadores pessoais ou de computadores no seu ambiente de trabalho. Foram realizadas discussões de dois casos clínicos: 1) relativo ao diagnóstico de câncer de mama, com a participação de quatro médicos; e 2) relativo ao acompanhamento de paciente internado em UTI com AVC, com a participação de três médicos.

As validações, realizadas com a participação de médicos especialistas, têm gerado subsídios para melhoria e otimização do ambiente, ressaltando que a etapa 4 encontra-se em progresso.

\section{Resultados e Discussões}

\section{Interfaces e funcionalidades do AVIIM}

A interface de acesso ao AVIIM, porta de entrada para o ambiente a partir da inserção de login e senha pelo usuário (médicos e demais profissionais cadastrados), apresenta, entre outros, um vídeo tutorial com informações sobre o ambiente.

As duas principais interfaces do sistema são: Instalação Caso Clínico e Discussão Caso Clínico, apresentadas a seguir.

\section{Interface - Instalação Caso Clínico}

Quando um médico deseja que determinado caso clínico seja analisado cooperativamente por outros médicos especialistas, pode cadastrar o mesmo no AVIIM e credenciar a equipe médica, caracterizando, assim, a instalação de um caso clínico para telediagnóstico.

A interface para Instalação de Caso Clínico (Figura 3) é composta por submódulos, quais sejam: Definir participantes (onde, em rotinas independentes, é realizada a definição do coordenador da equipe médica e a sua vinculação ao caso clínico); Inserir caso clínico (com rotinas específicas para a realização do relato do caso clínico e para a inserção dos resultados de exames - laboratoriais, complementares e outros - vinculados ao caso); Definir parâmetros (para o coordenador informar o período e o objetivo da equipe médica, de maneira que fique claro aos médicos participantes o que precisa ser discutido e qual o prazo para fazê-lo); Definir sessões (considerado como o principal submódulo deste módulo, disponibiliza ao coordenador da equipe médica as funcionalidades para criar e editar as sessões de trabalho, incorporando a cada sessão os recursos necessários para o desenvolvimento
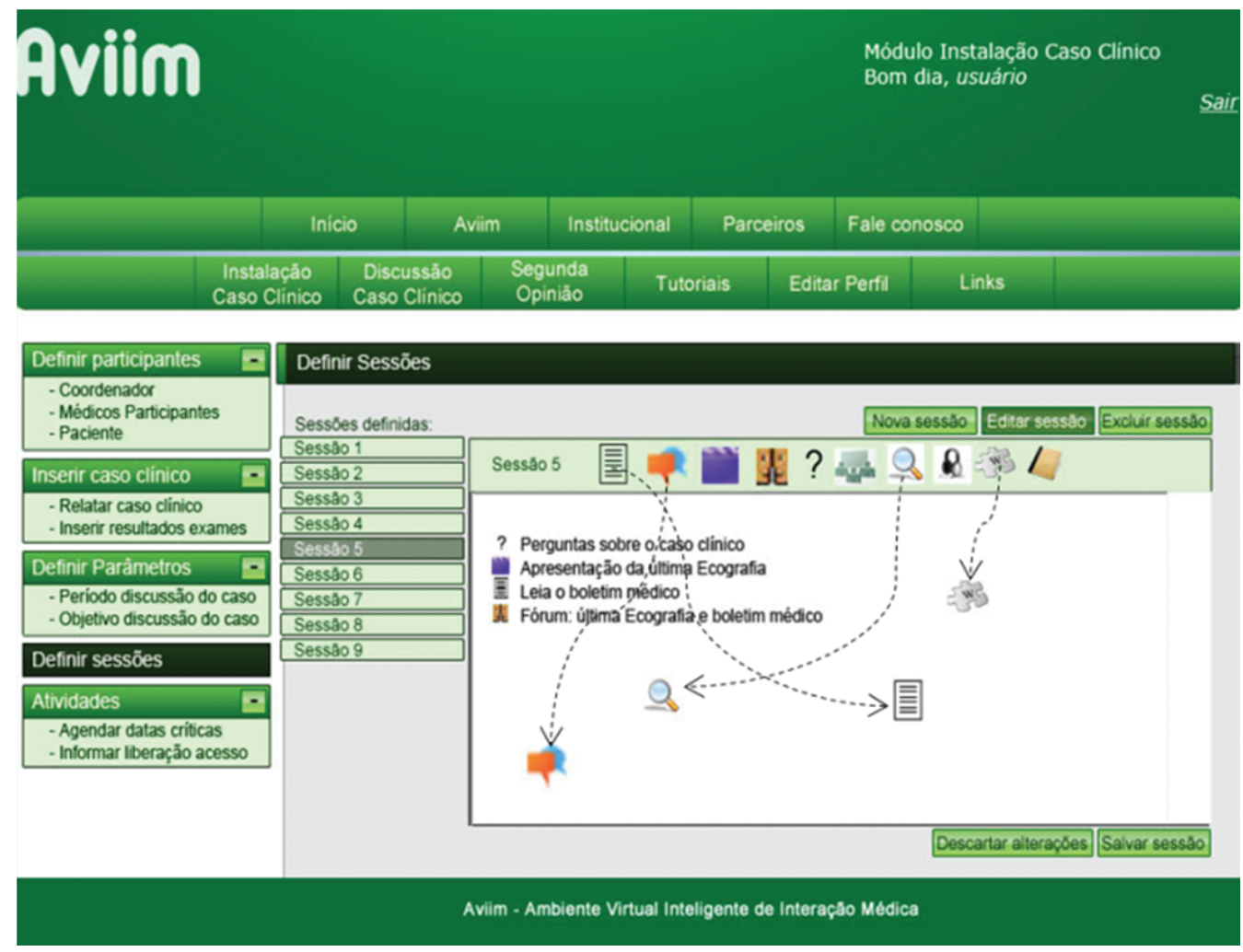

Figura 3. Interface instalação caso clínico.

Figure 3. Clinical case installation interface. 
dos trabalhos previstos); e Atividades (utilizado para a finalização da instalação da equipe médica, neste submódulo é realizado o agendamento das datas críticas e a liberação do acesso aos participantes, que recebem mensagem automática convidando-os a iniciar os trabalhos).

Ainda na Figura 3, dois exemplos de subtela (tela em nível interno) são apresentados: Definir Sessões (possibilita ao médico coordenador criar uma nova sessão e/ou editar e excluir sessões já cadastradas. Nesta subtela também é possível alterar a ordem de execução das sessões); e Editar Sessão (exemplificada com a edição da Sessão 5, onde ao usuário é possibilitado que inclua elementos/ferramentas na sessão apenas "arrastando" o elemento/ferramenta com o mouse para dentro da Sessão. Ao clicar em um elemento já incluído na sessão, é aberta uma subtela (nela deverão ser informados os parâmetros para execução daquele elemento/ferramenta).

Os dados de instalação do caso clínico e equipe médica são arquivados em uma única base de dados, que é utilizada conjuntamente pela equipe participante. Existem ferramentas específicas que permitem ao médico coordenador definir atividades, discussões e análises, com respectivos parâmetros e com datas e horários limites para realização, quando for o caso.

O fluxograma para o médico coordenador instalar um caso clínico é apresentado na Figura 4.

\section{Interface - Discussão caso clínico}

A interface para Discussão de Caso Clínico (Figura 5) é composta por submódulos, quais sejam: Busca (realização de pesquisa por palavras-chave no caso clínico em discussão e em outros casos clínicos em discussão ou arquivados, assim como em outros telediagnósticos realizados anteriormente e vinculadas à mesma instituição de saúde e/ou vinculadas a outras instituições, a partir de convênios realizados); Exames do pacientes (contendo os resultados dos exames laboratoriais, complementares e outros vinculados ao caso clínico); Pré-diagnósticos inteligentes AVIIM (índice de acesso direto aos MPDs disponíveis no AVIIM); Casos clínicos (lista de casos clínicos, subdividido em: i) Ativos, precedidos pela data de abertura do respectivo caso clínico; e ii) Banco de casos clínicos - com informações acessadas via pesquisa); Participantes (médico coordenador, médicos participantes, instituições às quais os

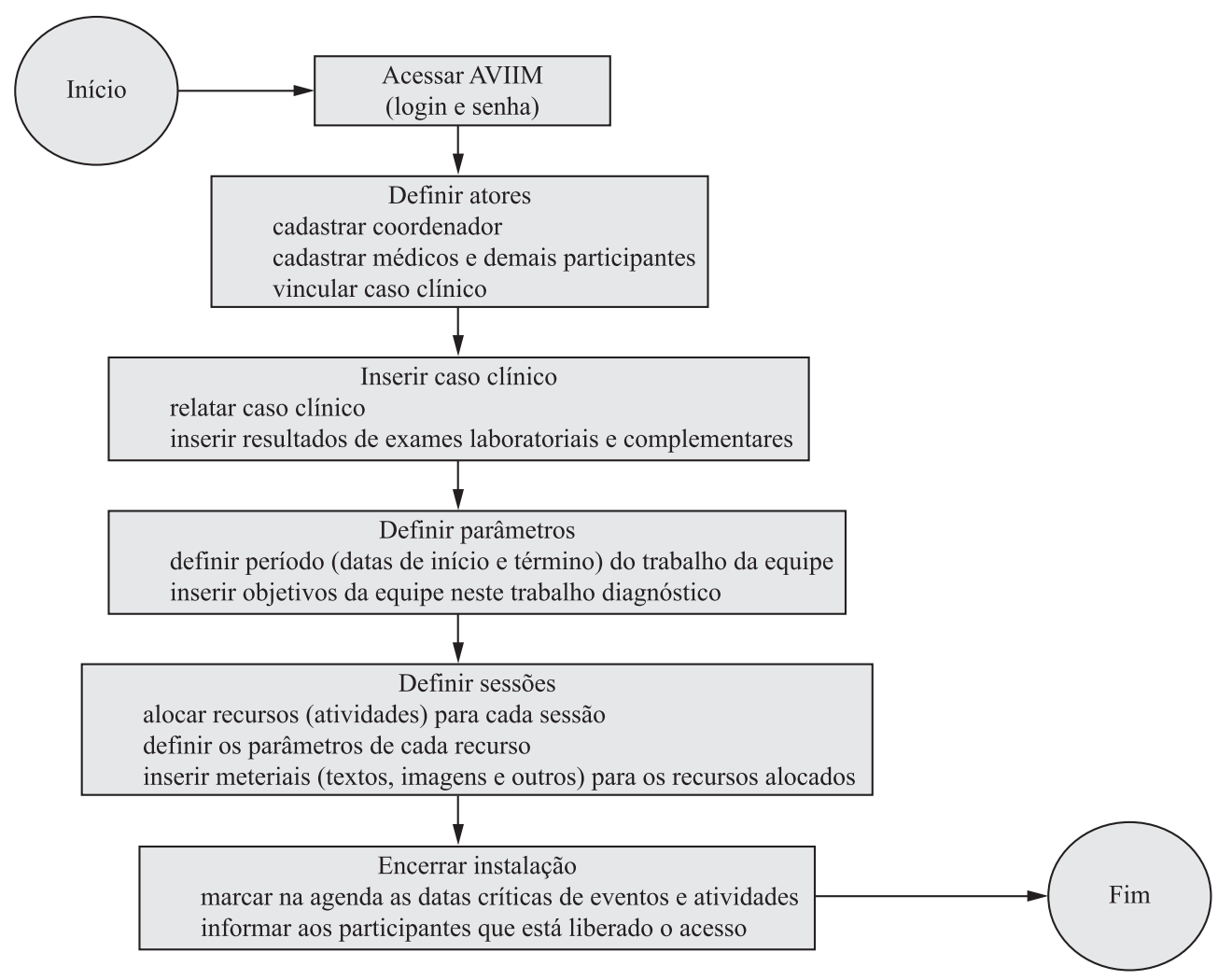

Figura 4. Fluxograma de instalação de caso clínico.

Figure 4. Clinical case installation flowchart. 


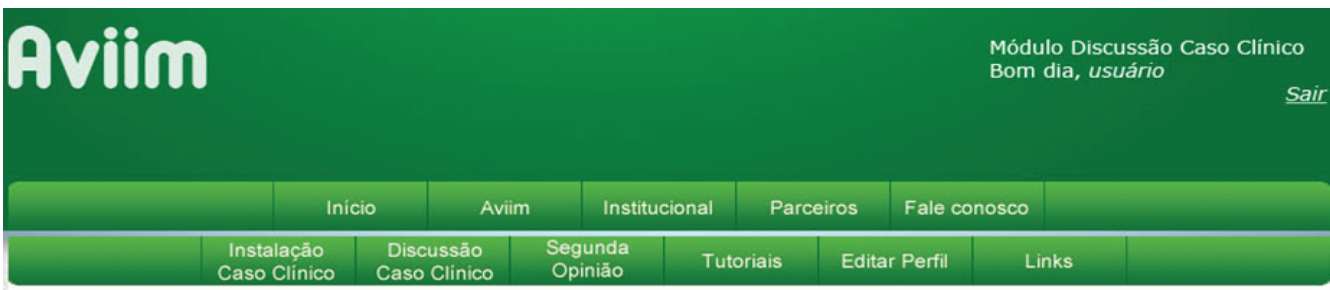

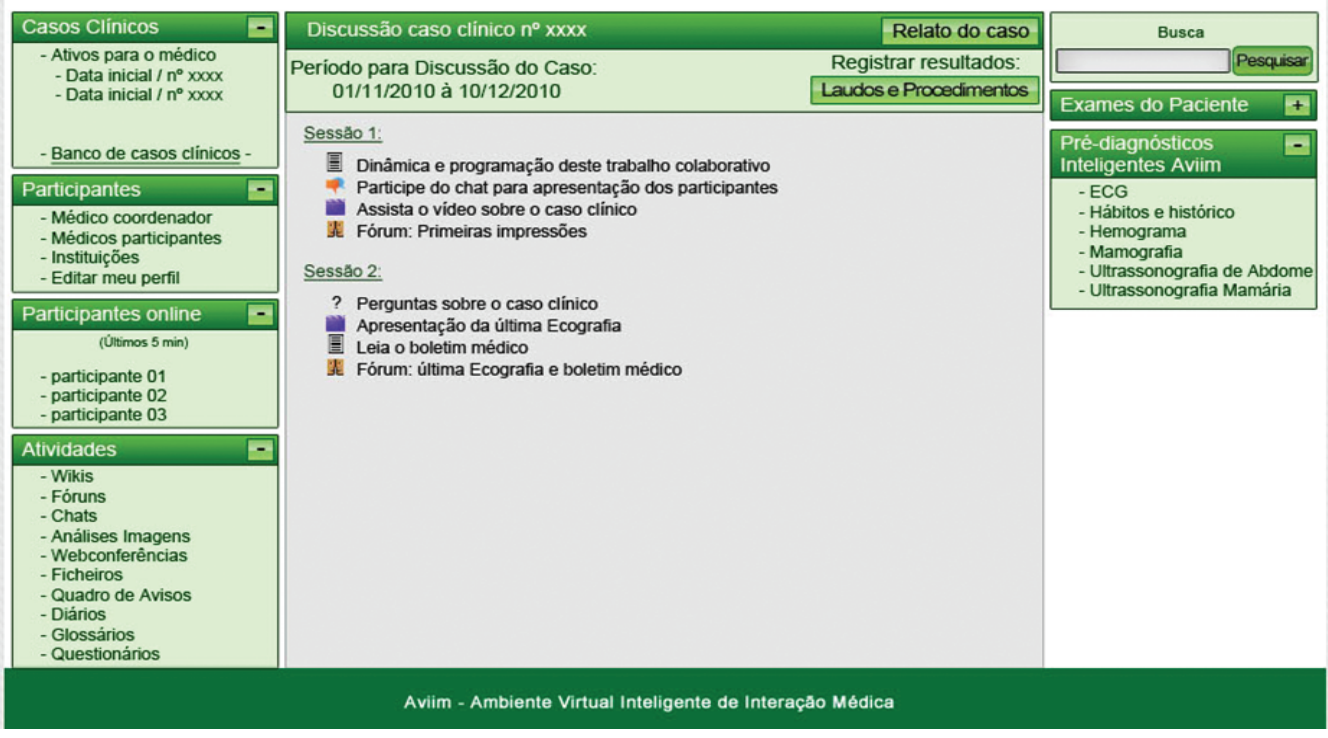

Figura 5. Interface discussão caso clínico.

Figura 5. Clinical case discussion interface.

participantes estão vinculados e edição do perfil); Participante online (lista de participantes ativos nos últimos $5 \mathrm{~min}$ ); Atividades (acesso direto às atividades de interação, tais como wikis, fóruns, chats, análises de imagens, webconferência, etc.);

No submódulo central - Discussão caso clínico $\mathbf{n}^{\mathbf{0}} \mathbf{x x x x}$ - são desenvolvidos os trabalhos da equipe médica, iniciando com o Relato do Caso e concluindo com os diagnósticos realizados Laudos e Procedimentos. As Sessões de trabalho são customizadas pelo médico coordenador a qualquer momento do processo, fazendo o direcionamento das discussões e demais atividades e sendo-lhe conferida total flexibilidade para organizar o material na página da forma que considerar mais conveniente. O coordenador pode, por exemplo, criar duas sessões para iniciar os trabalhos, conforme apresentado na Figura 5.

Relativo aos recursos que o AVIIM disponibiliza nesta interface para publicação, interação e avaliação, o médico coordenador dispõe de uma grande variedade e quantidade de ferramentas, quais sejam: disponibilização de informações e programação de atividades; páginas simples de texto; páginas em
HTML; acesso a arquivos em qualquer formato (pdf, doc, ppt, Flash, vídeo, etc.) ou a links externos (URLs); acesso a diretórios (pastas de arquivos no servidor); rótulos; atividades interativas com o AVIIM (Ex: solicitação de pré-diagnóstico a módulo especialista); livros eletrônicos; glossários; perguntas; análise de imagens, com interatividade síncrona e assíncrona; e questionários; chats; webconferência; wiki (para construção de textos de forma colaborativa); fórum de discussão; diários; ficheiro; mural. Também são disponibilizadas ferramentas para acompanhamento dos participantes: i) avaliação por acessos; e ii) avaliação por participação.

O funcionamento do AVIIM, na visão dos médicos participantes e considerando um caso clínico instalado para diagnóstico, é descrita a seguir e ilutrada na Figura 6.

Cada médico participante da equipe médica para telediagnóstico deve: i) acessar o AVIIM a partir de computador localizado em qualquer lugar, desde que conectado à internet, podendo o acesso ser realizado em qualquer horário para participação nas atividades assíncronas e em dia e hora pré-estabelecidos para participação em atividades síncronas; ii) conhecer o 


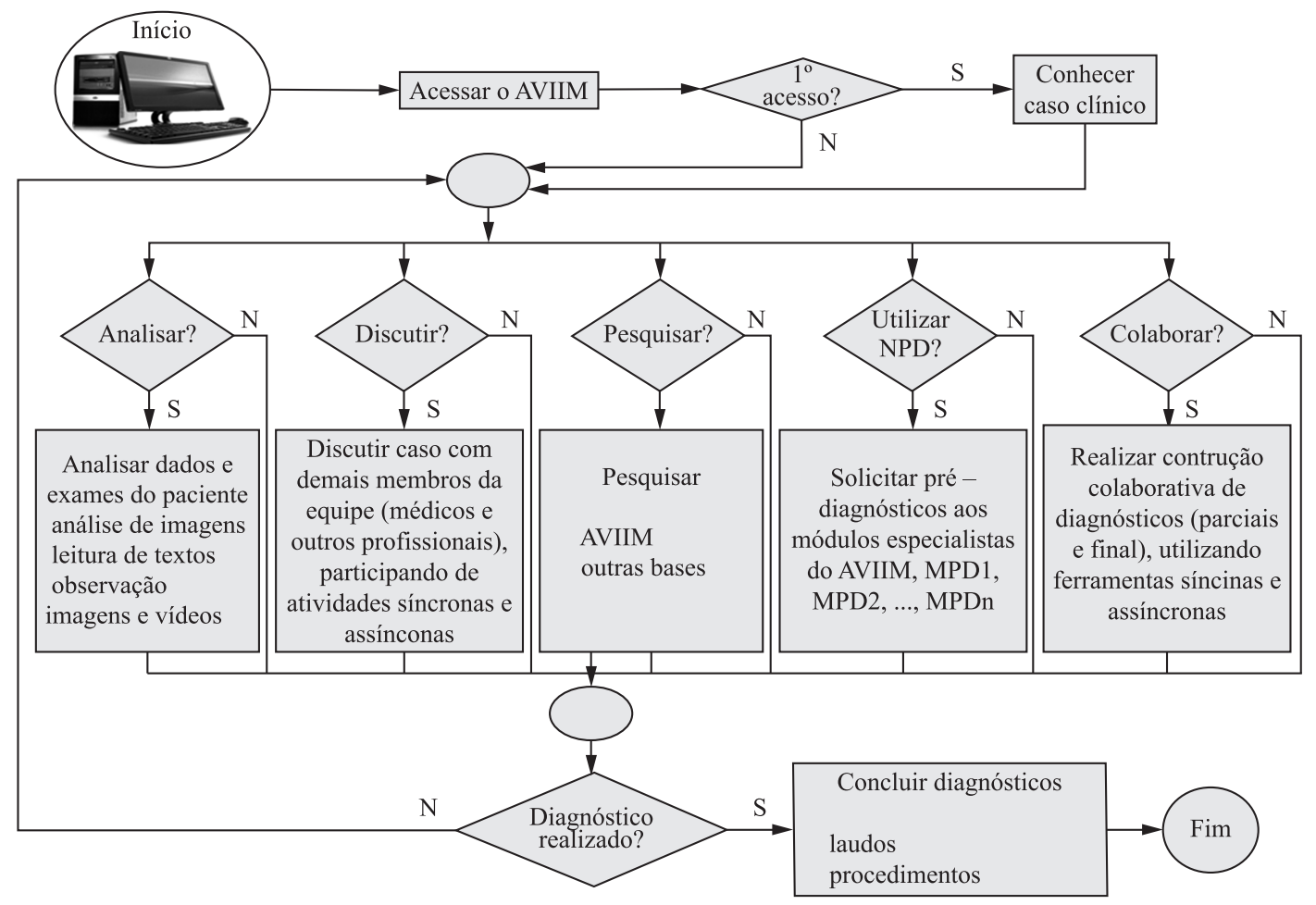

Figura 6. Fluxograma de discussão de caso clínico.

Figure 6. Clinical case discussion flowchart.

caso clínico (caso seja o primeiro acesso do médico), a partir do relato feito pelo médico coordenador; e iii) realizar as atividades previstas por esse médico em cada sessão e/ou aquelas disponíveis no ambiente que sejam de seu interesse, quais sejam: analisar dados (textuais ou não) e/ou resultados de exames (laboratoriais, complementares ou outros) do paciente, utilizando ferramentas para leitura de textos, observação e análise de imagens e observação de vídeo; discutir o caso clínico com os outros médicos, participando de atividades síncronas ou assíncronas; solicitar diagnósticos aos MPD do AVIIM; realizar construção cooperativa de diagnóstico(s); e concluir o diagnóstico final, a partir de trabalho colaborativo, emitindo o laudo conclusivo da equipe médica e a indicação dos procedimentos a serem adotados, no submódulo Laudos e Procedimentos.

\section{Conclusão}

O ambiente desenvolvido é uma solução computacional capaz de apoiar no diagnóstico médico, auxiliando a tomada de decisão, a partir da realização de trabalho colaborativo e utilizando técnicas de sistemas inteligentes. A utilização do AVIIM propicia a realização de telediagnóstico por equipes médicas ou multiprofissionais distantes geográficas e/ou temporalmente, além de possibilitar à equipe médica acesso a informações normalmente não disponíveis.

As principais contribuições deste trabalho são: a definição de um modelo de ambiente virtual de interação médica, customizado para a realização de telediagnóstico e segunda opinião; o projeto de interfaces especializadas (Instalação Caso Clínico e Discussão Caso Clínico); e a incorporação de componentes inteligentes a um ambiente virtual para auxílio ao telediagnóstico médico.

\section{Agradecimentos}

Ao Conselho Nacional de Desenvolvimento Científico e Tecnológico (CNPq), ao Hospital Promater, ao Laboratório de Sistemas Inteligentes (LABSIS) e ao Laboratório de Automação Hospitalar e Bioengenharia (LAHB) da Universidade Federal do Rio Grande do Norte (UFRN).

\section{Referências}

Barbosa AKP, Novaes MA, Vasconcelos AML. A web application to support telemedicine services in Brazil. In: AMIA Simposium: Proceedings of the AMIA Simposium; 2003 nov 8-12; Washington, USA. Washington; 2003. p. 56-60. 
Encyclopedia of Educational Technology - EET. 2009. [cited 2009 nov 09]. Available from: http://coe.sdsu.edu/eet/

Engelbrech AP. Computational Intelligence: an introduction. 2th ed. Chichester: John Wiley \& Sons Ltd; 2007.

European Commission. Telemedicine for the benefit of patientes, healthcare systems and society. Brussels: European Commission; 2009.

Feng DD. Biomedical Information Technology. Hardcover: Academic Press; 2007. Series-Academic Press Series in Biomedical Engineering.

Health Level Seven Standards - HL7. Health Level Seven International. 2010. [cited 2010 jan 19]. Available from: http://www.hl7.org

Hovenga EJS, Kidd MR, Garde S, Cossio CHL. Health Informatics: An Overview. Amsterdam: IOS Press; 2010.

Krsek P, Spanel M, Svub M, Stancl V, Siler O, Barton R. Network collaborative environment supporting 3D medicine. In: EMBC 2009: Proceedings of the Annual International Conference of the IEEE Engineering in Medicine and Biology Society; 2009 Sept 3-6; Minneapolis, MN, USA. Minneapolis: EMBC; 2009. p. 2164-7.

Leite CRM, Martins DL, Sizilio GRMA, Santos KEA, Araújo BG, Valentim RAM, Dória-Neto AD, Melo JD, Guerreiro AMG. Classification of cardiac arrhythmias using competitive networks. In: EMBC2010: Proceedings of the 32nd Annual International Conference of the IEEE Engineering in Medicine and Biology Society; 2010 August 31-September 4; Buenos Aires, Argentina. Buenos Aires: EMBC; 2010. p. 1386-9.

Organização Mundial de Saúde - OMS. 2009. [cited 2009 aug 18]. Available from: http://www.who.org
Paulon SM, Carneiro MLF. A educação a distância como dispositivo de fomento às redes de cuidado em saúde. Interface: Comunicação, Saúde e Educação. 2009; 13:747-58.

Sabbatini RME, Costa CGA, César S, Cardoso SH, Perdigão VLM. Rede São Paulo Saudável: Um sistema de educação profissional à distância para a saúde pública, baseado em satélite digital e internet. In: Congresso Brasileiro de Informática em Saúde: Anais do IX Congresso Brasileiro de Informática em Saúde; 2010 out; São Paulo. São Paulo: Sociedade Brasileira de Informática em Saúde; 2010.

Sarhan F. Telemedicine in healthcare 2: the legal and ethical aspects of using new technology. Nursing Times. 2009; 105(43):18-20.

Sociedade Brasileira de Informática em Saúde - SBIS. Manual de Certificação para Sistemas de Registro Eletrônico em Saúde. SBIS; 2009. v. 3.3.

Shen Y, Xi N, Methil-Sudhakaran N, Mukherjee R, Zhu D, Cen Z, Mutka MW, Slomski CA, Apelgren KN. Internet based Tele-Diagnostic Interface for Breast Pathology. In: 564 Telehealth: Proceedings of the 564 Telehealth; 2007 may 31- Jun 1; Montreal, QC, Canada. Montreal; 2007.

Soares AMP. Um ambiente de suporte ao trabalho cooperativo com aplicação à telemedicina [dissertação]. Natal: Universidade Federal do Rio Grande do Norte; 2002.

Soares HB. Análise e Classificação de Imagens de Lesões da Pele por Atributos de Cor, Forma e Textura utilizando Máquina de Vetor de Suporte [tese]. Natal: Universidade Federal do Rio Grande do Norte; 2008.

Sriraam N, Eswaran C. Performance evaluation of neural network and linear predictors for near-lossless compression of EEG signals. IEEE Transactions on Information Technology in Biomedicine. 2008; 12:87-93. PMid:18270040. http://dx.doi.org/10.1109/TITB.2007.899497

\footnotetext{
Autores

Gláucia Regina Medeiros Azambuja Sizilio, Ana Maria Guimarães Guerreiro, Adrião Duarte Dória Neto Departamento de Engenharia da Computação e Automação - DCA, Laboratório de Sistemas Inteligentes - LABSIS, Universidade Federal do Rio Grande do Norte - UFRN, Av. Senador Salgado Filho, 3000, Lagoa Nova, CEP 59078-970, Natal, RN, Brasil
} 